Mathematical Modelling and Analysis

Volume 16 Number 3, September 2011, 442-450

Doi:10.3846/13926292.2011.602987

(C) Vilnius Gediminas Technical University, 2011
Publisher: Taylor\&Francis and VGTU

www.tandfonline.com/loi/tmma20

Online ISSN: 1648-3510

Print ISSN: 1392-6292

\title{
Potential Representation Method for the Schrödinger Equation
}

\section{Arvydas Juozapas Janavičius, Donatas Jurgaitis and Sigita Turskienè}

\author{
Šiauliai University \\ P. Višinskio st. 19, LT-77156 Šiauliai, Lithuania \\ E-mail: AYanavy@gmail.com; pletra@cr.su.lt \\ E-mail(corresp.): sigita@fm.su.lt
}

Received June 22, 2010; revised May 19, 2011; published online August 1, 2011

\begin{abstract}
A general solution of the Schrödinger equation in the potential representation has been obtained in the form of integral equations. In this representation, the wave function for positive and negative energies or bound states can be expressed as a product of the unperturbed solution for model potential and the function which depends on the additional potential or potential perturbation. Here we have proved that this method is equivalent to the method of variation of constants for negative energies. The linearly independent solutions of Schrödinger equation for harmonic oscillator potential have been obtained for derivation of integral equations, which are used for finding eigenfunctions and eigenvalues for Woods-Saxon potential. Eigenvalues obtained by numerical iterations of these integral equations are in good agreement with results obtained by the discretization method. The kernels of the obtained integral equations are proportional to the perturbation or difference of Woods-Saxon and harmonic oscillator potentials.
\end{abstract}

Keywords: Schrödinger equation, Green's function, integral equation, boundary value problem, iterative method.

AMS Subject Classification: 34L10; 34L15.

\section{Introduction}

We have obtained the analytical solutions of the one-particle radial Schrödinger equation for negative energies in the form of the integral equations by using the potential representation method proposed for positive [8, 9, 10], and negative [3] energies. The main idea of this method is expressing the radial wave function as a product of the model solution and the function, which depends on the difference of interaction potentials. This paper is a generalization of integral equations derived in [10] for positive energies, where the radial wave functions can be obtained by multiplying the free solution on function which depends on the perturbation potential. In [10], the following postulate was presented: after 
adding potential to Hamiltonian, a new radial wave function can be obtained multiplying unperturbed or model wave function on function which depends on added potential. We have proved this postulate $[3,4,9]$ and presented it like a theorem using the method of indefinite coefficients. From this theorem, the connection between the solutions for short range potentials and for Coulomb potentials has been obtained [9]. In potential representation $[2,3,4,15]$ the perturbed radial function $U_{\alpha n L}(r)$ with the set of quantum numbers $\alpha n L$ can be expressed as a product of unperturbed wave function $U_{0 n L}(r)$ with the set of quantum numbers $n L$

$$
U_{\alpha n L}(r)=\varphi_{\alpha}(r) U_{0 n L}(r)
$$

on the multiplier $\varphi_{\alpha}(r)$ depending on an additional potential. The main problem considered in this paper is the interpretation and convergence of the obtained eigenvalues using the harmonic oscillator potential for modelling of Woods-Saxon potential. At large distances these potentials have essentially different asymptotical behaviour, i.e. $r^{2}$ and $\exp [-\alpha r], \alpha>0$, respectively.

\section{Modelling of the Schrödinger Equation Solutions with the Harmonic Oscillator Potential}

We consider the Schrödinger equation

$$
\frac{d^{2}}{d r^{2}} U_{\alpha n L}-\frac{L(L+1)}{r^{2}}+c\left[E_{0 n L}+\Delta E_{\alpha}-V_{D}(r)-V_{0}(r)\right] U_{\alpha n L}=0
$$

where $c=2 \mu / \hbar^{2}$, and potentials are defined as

$$
\begin{aligned}
& V_{0}(r)=\frac{m \omega^{2} r^{2}}{2}-V_{S}(0), \quad V_{D}(r)=V_{S}(r)-V_{0}(r), \\
& V_{S}(r)=-V\left[1+\exp [\alpha(r-R)]^{-1} .\right.
\end{aligned}
$$

Here we have added and subtracted the potential $V_{0}(r)$. The energies are expressed by the sum of energies $E_{0 n L}$ for a model potential and energy changes $\Delta E_{\alpha}$ for perturbation $V_{D}(r)$. We are interested in finding eigenvalues $E_{0 n L}+$ $\Delta E_{\alpha}$ and eigenfunctions $U_{\alpha n L}$ of the equation (2.1) with perturbed potential $V_{D}(r)$ by using unperturbed analytical physical $U_{0 n L}$ and linearly independent nonphysical $F_{0 n L}$ solutions and eigenvalues $E_{0 n L}$ of the Schrödinger equation for model potential $V_{0}(r)$. For the harmonic oscillator potential, we have obtained the following solutions [3]:

$$
\begin{aligned}
& U_{0 n L}=e^{-0.5 \rho} \rho^{0.5(L+1)} \sum_{k=0}^{n-1} a_{k} \rho^{k}, \quad \rho=\frac{m \omega r^{2}}{\hbar}, \quad n=1,2,3, \\
& a_{k+1}=\frac{k-0.5\left(\varepsilon_{n L}-L-1.5\right)}{(k+1)(k+L+1.5)} a_{k}, \quad a_{0}=1,
\end{aligned}
$$




$$
\begin{aligned}
& F_{0 n L}=e^{-0.5 \rho} \rho^{-0.5 L} w(\rho), \quad w(\rho)=\sum_{k=0}^{\infty} b_{k} \rho^{k}, \\
& b_{k+1}=\frac{k-0.5\left(\varepsilon_{n L}+L-0.5\right)}{(k+1)(k-L+0.5)} b_{k}, \quad b_{0}=1,
\end{aligned}
$$

and eigenvalues

$$
\begin{aligned}
& E_{0 n L}=\varepsilon_{n L} \hbar \omega-V_{S}(0), \quad \varepsilon_{n L}=2 n+L-0.5, \\
& \omega=d \omega_{0}, \quad \omega_{0}=41 A^{-1 / 3} \frac{M e V}{\hbar} .
\end{aligned}
$$

Frequencies $\omega_{0}$ for harmonic oscillator potential can be determined [3] from the radius of nuclei $R$ and depend on nucleon number $A$ and $V_{s}(0)=-V$ from (2.2). The coefficients of power series $w(\rho)$ for very high powers satisfy the abbreviated recursion relation

$$
b_{k+1} / b_{k} \rightarrow 1 / k, \quad k \gg 1 .
$$

From this ratio and $[4,12]$ and $(2.3)$ we can make a conclusion that for $\rho \rightarrow \infty$ we have

$$
w(\rho) \rightarrow \mathrm{e}^{\rho}, \quad F_{0 n L} \rightarrow \mathrm{e}^{0.5 \rho} .
$$

From the asymptotical expressions $\lim _{\rho \rightarrow 0} U_{0 n L}=\rho^{L+1}$ and $\lim _{\rho \rightarrow 0} F_{0 n L}=$ $\rho^{-L}$ we can obtain the Wronskian [3]

$$
W_{0}\left(F_{0 n L}, U_{0 n L}\right)=F_{0 n L} \frac{d U_{0 n L}}{d r}-U_{0 n L} \frac{d F_{0 n L}}{d r}, \quad W_{0}=(2 L+1)\left(\frac{m \omega}{\hbar}\right)^{\frac{1}{2}} .
$$

The perturbed $U_{\alpha n L}$ and unperturbed $U_{0 n L}$ solutions must have the same boundary condition at the origin $r^{L+1}$ and at the infinity:

$$
\lim _{r \rightarrow 0} \varphi_{\alpha}=1, \quad \lim _{r \rightarrow \infty} \varphi_{\alpha}(r) U_{0 n L}(r)=0 .
$$

\section{Green's Functions for the Potential Representation}

The multiplicative or potential representation perturbation theory can be realized by using the modified method of Lagrange $[2,4,15]$. In this case perturbed solution $U_{\alpha n L}$ of equation (2.1) for perturbation potential $V_{D}(r)$ can be expressed in the following way

$$
U_{\alpha n L}=C_{1}(r) F_{0 n L}(r)+C_{2}(r) U_{0 n L}(r),
$$

where $F_{0 n L}(r)$ and $U_{0 n L}(r)$ are linearly independent solutions for model potential $V_{0}(r)$. Using the same factorization like in [15]

$$
U_{\alpha n L}=\left[C_{1}(r) \frac{F_{0 n L}(r)}{U_{0 n L}(r)}+C_{2}(r)\right] U_{0 n L}(r), \quad U_{\alpha n L}(r)=\varphi_{\alpha} U_{0 n L}(r),
$$

and the additional condition [15] for derivatives of indefinite coefficients

$$
C^{\prime}{ }_{1}(r) \frac{F_{0 n L}(r)}{U_{0 n L}(r)}+C^{\prime}{ }_{2}(r)=0
$$


we obtain derivatives

$$
\begin{aligned}
\varphi^{\prime}{ }_{\alpha} & =-W_{0} \frac{C_{1}(r)}{U_{0 n L}{ }^{2}(r)}, \quad W_{0}=F_{0 n L} U^{\prime}{ }_{0 n L}-U_{0 n L} F_{0 n L}^{\prime}, \\
\varphi^{\prime \prime}{ }_{\alpha} & =-W_{0}\left[\frac{C_{1}^{\prime}(r)}{U_{0 n L}{ }^{2}(r)}-2 C_{1}(r) \frac{U_{0 n L}^{\prime}(r)}{U_{0 n L}{ }^{3}(r)}\right] .
\end{aligned}
$$

Here $W_{0}$ is Wronskian of linearly independent solutions $F_{0 n L}(\mathrm{r})$ and $U_{0 n L}$. Substituting (3.2), (3.4) and (3.5) into (2.1) and including expression (3.3) we get

$$
\begin{aligned}
& C_{1 \alpha}^{\prime}(r)=-\frac{1}{W_{0}} U_{0 n L} c V_{d} \varphi_{\alpha} U_{0 n L}, \quad V_{d}=V_{D}-\Delta E_{\alpha} \\
& C_{2 \alpha}^{\prime}(r)=\frac{1}{W_{0}} F_{0 n L} c V_{d} \varphi_{\alpha} U_{0 n L} .
\end{aligned}
$$

Integrating the last equation and taking into account boundary conditions (2.10) we obtain

$$
\begin{aligned}
& C_{1 \alpha}(r)=-\frac{1}{W_{0}} \int_{0}^{r} U_{0 n L} c V_{d} \varphi_{\alpha} U_{0 n L} d r_{1} \\
& C_{2 \alpha}(r)=\beta_{\alpha}-\frac{1}{W_{0}} \int_{r}^{\infty} F_{0 n L} c V_{d} \varphi_{\alpha} U_{0 n L} d r_{1} \\
& \beta_{\alpha}=1+\frac{1}{W_{0}} \int_{0}^{\infty} F_{0 n L} c V_{d} \varphi_{\alpha} U_{0 n L} d r_{1} .
\end{aligned}
$$

Substituting these equations into (3.1) we obtain the integral equation for perturbed eigenfunctions

$$
\begin{aligned}
\varphi_{\alpha} U_{0 n L}= & U_{0 n L} \beta_{\alpha}-\frac{F_{0 n L}}{W_{0}} \int_{0}^{r} U_{0 n L} c V_{d} \varphi_{\alpha} U_{0 n L} d r_{1} \\
& -\frac{U_{0 n L}}{W_{0}} \int_{r}^{\infty} F_{0 n L} U_{0 n L} c V_{d} \varphi_{\alpha} U_{0 n L} d r_{1}
\end{aligned}
$$

The presented equation can be rewritten as

$$
\varphi_{\alpha} U_{0 n L}=U_{0 n L} \beta_{\alpha}+\int_{0}^{\infty} G_{0}\left(r_{>}, r_{<}\right) V_{d} \varphi_{\alpha} U_{0 n L} d r_{1},
$$

where according to [9] the kernel of this integral equation is Green's function

$$
G_{0}=-F_{0 n L}\left(r_{>}\right) U_{0 n L}\left(r_{<}\right) / W_{0} .
$$

Using (3.8) we can reduce equation (3.9) into a more convenient form

$$
\begin{aligned}
& \varphi_{\alpha} U_{0 n L}=U_{0 n L}+\frac{U_{0 n L}}{W_{0}} \int_{0}^{r} F_{0 n L} c V_{d} \varphi_{\alpha} U_{0 n L} d r_{1}-\frac{F_{0 n L}}{W_{0}} \int_{0}^{r} U_{0 n L} c V_{d} \varphi_{\alpha} U_{0 n L} d r_{1}, \\
& V_{d}(r)=V_{S}(r)-V_{0}(r)-\Delta E_{\alpha} .
\end{aligned}
$$


Here we have the kernels of integral equation

$$
M_{1}(r)=F_{0 n L}(r) V_{d}(r), \quad M_{2}(r)=U_{0 n L}(r) V_{d}(r) .
$$

Requiring that perturbed solution $\varphi_{\alpha} U_{0 n L}$ would satisfy boundary condition (2.10) and taking in into account (2.8), we obtain the following expression for calculation of perturbed eigenvalues at $N$-th iteration

$$
\begin{aligned}
& E_{\alpha n L N}=E_{0 n L}+\Delta E_{\alpha N}, \quad \Delta E_{\alpha N}=\frac{\int_{0}^{\infty} U_{0 n L} V_{D}(r) \varphi_{\alpha N} U_{0 n L} d r}{\int_{0}^{\infty} U_{0 n L} \varphi_{\alpha} U_{n L} d r}, \\
& V_{D}(r)=V_{S}(r)-V_{0}(r) .
\end{aligned}
$$

The integral equations (3.11), (3.13) can be solved by the iteration method. For the first iteration of $\Delta E_{\alpha n L 0}$ and $\varphi_{\alpha 0} U_{0 n L}$ in the integrals (3.13) and the right hand side of (3.11) we must take $\varphi_{\alpha 0}=1, N=0$. For calculation of more exact values of energy $E_{\alpha n L}$ and perturbed eigenfunctions, we must solve integral equations using the obtained values of $\Delta E_{\alpha 0}$ and $\varphi_{\alpha 1} U_{0 n L}$.

\section{The Accuracy of Obtained Solutions}

Theorem 1. The changes $\Delta E_{\alpha}$ of the unperturbed eigenvalues $E_{0 n L}$ and potential representation functions $\varphi_{\alpha}$ depend on perturbation of potential energy $V_{d}$ and weakly depend on the parameter of the integral equation.

Proof. Convergence of eigenvalues $E_{\alpha n L}$ depends on absolute values of kernels (3.12) of the integral equation (3.11). For that consideration, we can present the integral equation (3.11) in the following way

$$
\begin{aligned}
\varphi_{\alpha} U_{0 n L}= & U_{0 n L}+\lambda\left[U_{0 n L} \int_{0}^{z} F_{0 n L} V_{d} \varphi_{\alpha} U_{0 n L} d z_{1}\right. \\
& \left.-F_{0 n L} \int_{0}^{z} U_{0 n L} c V_{d} \varphi_{\alpha} U_{0 n L} d z_{1}\right], \quad \lambda=\frac{c F^{2}}{W_{0}},
\end{aligned}
$$

where we have introduced new dimensionless variable $z$ expressed by $F$-Fermi unite $10^{-15} \mathrm{~m}$. From (2.7), (2.9) we calculate values of parameters $\lambda_{1}=$ $0.73701 \cdot 10^{-3} / \sqrt{d}$ and $\lambda_{2}=0.79991 \cdot 10^{-3} / \sqrt{d}$ of integral equation (4.1) for neutrons of nucleus $A_{1}=208$ and $A_{2}=340$. Taking into account that $d \in[0.6 ; 1.2]$ we can use the method proposed in [14], where the solution of integral equation can be expressed by power series of $\lambda$. Using this method, we suppose that for sufficiently large number $N$ of iterations we can present approximately

$$
\varphi_{\alpha}=\sum_{m=0}^{N} \varphi_{m \alpha} \lambda^{m}, \quad \varphi_{0 \alpha}=1
$$

For the first approach we take in (3.13) $\varphi_{0 \alpha}=1$ and energy improvement (3.13) $\Delta E_{\alpha 0}$ not depending on $\lambda$ we obtain $1+\varphi_{1 \alpha} \lambda$. Then we obtain that $\varphi_{1 \alpha}$ does not depend on $\lambda$

$$
\lambda \varphi_{1 \alpha} U_{0 n L}=\lambda\left[U_{0 n L} \int_{0}^{z} F_{0 n L} V_{d 0} U_{0 n L} d z_{1}-F_{0 n L} \int_{0}^{z} U_{0 n L} V_{d 0} U_{0 n L} d z_{1}\right],
$$


but depends only on differences $V_{d N}(r)=V_{S}(r)-V_{0}(r)-\Delta E_{\alpha, N}, V_{0}(r)=$ $m \omega^{2} r^{2} / 2-V_{S}(0)$ of the Woods-Saxon $(2.2) V_{S}(r)$ and model $V_{0}(r)$ potentials for variable parameter $\omega=d \omega_{0}$. The $U_{0 n L}$ and $F_{0 n L}$ are physical and nonphysical solutions [3] of Schrödinger equation for the model potential. Substituting (4.2) into (4.1) and (3.13) for any iterations $N$ we obtain

$$
\begin{aligned}
& \varphi_{N+1, \alpha} U_{0 n L}=U_{0 n L} \int_{0}^{r} F_{0 n L} V_{d N} \varphi_{N \alpha} U_{0 n L} d z-F_{0 n L} \int_{0}^{r} U_{0 n L} V_{d N} \varphi_{N \alpha} U_{0 n L} d z \\
& V_{d N}(r)=V_{S}(r)-V_{0}(r)-\Delta E_{\alpha, N} \\
& E_{\alpha, N}=\int_{0}^{\infty} U_{0 n L} V_{D} U_{0 n L} d r+\lambda \int_{0}^{\infty} U_{0 n L} V_{D} \varphi_{1 \alpha} U_{0 n L} d r \\
& \quad+\cdots \lambda^{N} \int_{0}^{\infty} U_{0 n L} V_{D} \varphi_{N \alpha} U_{0 n L} d r
\end{aligned}
$$

It is well known in quantum mechanics that the first iteration for $\Delta E_{\alpha}$ of perturbation calculations is the most important for a small perturbation [11]. Providing variation of $d$ or $\omega$ we can solve integral equations (3.13), (4.1) for the small potential perturbation $V_{D}=V_{S}-V_{0}$ in the region $z \in\left[0 ; R_{m}\right]$ with the minimum number of iterations $N$ for definite precisely. The second iteration for $\Delta E_{\alpha}$ will be obtained substituting in (3.13) approximate function $\varphi_{\alpha}=$ $1+\lambda \varphi_{1 \alpha}$ depending on $\lambda(4.1),(2.7)$ or $\sqrt{d}$. We have the kernels (3.12) of integral equation (4.1) where perturbations of the energies $\Delta E_{\alpha}$ depending on quantum states are included. In this case, the optimal values of parameter $d$ must be different for different quantum states or energy levels $E_{\alpha n L}$.

In [14], it was proved that solutions of integral equations like (4.1) converge uniformly, when the following conditions

$$
\left|U_{0 n L}\right|<N_{U}, \quad U_{0 n L} \int_{0}^{R_{m}} F_{0 n L} V_{d} d z-F_{0 n L} \int_{0}^{R_{m}} U_{0 n L} V_{d N} d z<M,
$$

are satisfied. Thus in our case, we should prove that the following conditions

$$
\left|\varphi_{m \alpha} U_{0 n L}\right|<N_{U} M^{m}, \quad|\lambda|<\frac{1}{M}-\varepsilon \quad(\varepsilon>0),
$$

are satisfied. We can normalize [5] the eigenfunctions $U_{0 n L}$ and then we have in (4.5) that $N_{U}<1$. Theoretical evaluation of $M$ is a hard task, but experimentally we have obtained the convergence of eigenvalues (3.13), (4.4) $\Delta E_{\alpha n L N}$ and eigenfunctions (4.1) $\varphi_{\alpha} U_{0 n L}$ for $\frac{0.73701 \cdot 10^{-3}}{\sqrt{d}} \leq \lambda \leq \frac{0.79991 \cdot 10^{-3}}{\sqrt{d}}$ when $d \in[0.6 ; 1.2]$. For small values of $\omega$ or parameter $d$ the eigenvectors $E_{\alpha n L}$ depend on perturbation $V_{d}$ and very weakly on $\lambda$, parameter $M$ has a finite value. Theorem 1 is proved.

It follows that model potential $V_{0}(r)$ for $r \rightarrow R_{m}$ is increasing like $r^{2}$ but $U_{0 n L}$ are rapidly decreasing like $\exp \left(-C r^{2}\right)$. We have a convergence of eigenvalues $E_{\alpha n L}$ and eigenfunctions for equations (3.13) and (4.1) at large distances $R_{m}$ when Theorem 1 is satisfied and eigenvalues weakly depend on parameter $\lambda$. 
In computational experiments we have used different limits of integration $R_{m}$ in (3.13), (4.1) from 0 till $1.5 R$ or $2.4 R$, where $R=1.24 A^{1 / 3}$ is a nucleus radius expressed in Fermi units. Integration limits are increasing for decreasing energies of nucleons $\left|E_{\alpha n L}\right|$ [4]. In order to get approximate solutions we must find eigenvalues which only weakly depend on $d$ in the some region of $\Delta d$, because the exact eigenvalues do not depend on the parameters $\omega$ of model potential. Practically, we can find approximate solutions by changing $\omega=d \omega_{0}$ in the interval $d \in[0.6 ; 1.2]$ [3]. We must choose solutions giving minimum values of $\left|\Delta E_{\alpha n L}\right|$ and minimum iterations numbers for calculation of eigenvalues with some given accuracy.

In [4], calculations of energies $E_{n L j}$ were done for Woods-Saxon potential of one-nucleon energy levels for neutrons of nucleus ${ }^{197} \mathrm{Au}$. Integral equations were solved with the variation of parameters $d$ and $\omega$ of the model potential. The energies $E_{n L j}$ expressed in $\mathrm{MeV}$ (Mega-electron-Volt) were compared with the eigenvalues $\bar{E}_{n L j}$ obtained by the discretization method, in the interval $14 \mathrm{fm}\left(10^{-15} \mathrm{~m}\right)$ with the step $0.2 \mathrm{fm}$ by using program EIGEN [13]. Results for quantum states with principal quantum numbers $n$, orbital-momentum quantum numbers $L=0$ ( $s$ state) and resulting momentum quantum numbers $j=1 / 2$ are presented in Table 1 . Here $N$ is the number of iterations.

Table 1. The energy levels $\bar{E}_{n L j}$ and $E_{n L j}$ of neutrons of nucleus ${ }^{197} \mathrm{Au}$.

\begin{tabular}{lllll}
\hline$n L j$ & $\bar{E}_{n L j}, \mathrm{MeV}$ & $E_{n L j}, \mathrm{MeV}$ & $d$ & $N$ \\
\hline $1 s_{1 / 2}$ & -39.03 & -39.36 & 1.00 & 6 \\
$2 s_{1 / 2}$ & -28.05 & -28.42 & 1.00 & 14 \\
$3 s_{1 / 2}$ & -13.42 & -13.98 & 1.00 & 18 \\
\hline
\end{tabular}

The same energy levels were calculated with program EIGEN [13] and by solving integral equations (3.12), (4.1), with the parameter $d$ and $\omega$ of the model potential (2.2) for nucleus ${ }^{208} \mathrm{~Pb}$. The results are presented in Table 2.

Table 2. The energy levels $\bar{E}_{n L j}$ and $E_{n L j}$ of neutrons of nucleus ${ }^{197} \mathrm{~Pb}$.

\begin{tabular}{lllll}
\hline$n L j$ & $\bar{E}_{n L j}, \mathrm{MeV}$ & $E_{n L j}, \mathrm{MeV}$ & $d$ & $N$ \\
\hline $1 s_{1 / 2}$ & -39.97 & -39.93 & 0.67 & 3 \\
$2 s_{1 / 2}$ & -29.56 & -29.48 & 0.83 & 13 \\
$3 s_{1 / 2}$ & -15.38 & -15.32 & 0.82 & 17 \\
\hline
\end{tabular}

\section{Conclusions}

Comparing the energy levels presented in Tables 1 and 2, we see that using the variation of the parameter $d$ of model potential, the significantly better coincidence of eigenvalues with the control calculations using standard program 
EIGEN [13] has been obtained. We found that results obtained solving integral equations (3.13), (4.1) weakly depend on the parameter $d$ or $\omega$ of the model potential for a minimum number of iterations $N$, if the step and limits of integration $R_{m}$ are chosen correctly.

Here we have proposed a new method and derived integral equations for negative energies, where the expansion of perturbed eigenfunctions by infinite number of unperturbed eigenfunctions [1] can be avoided. We have used previously obtained [3] linearly independent solutions for finding Green functions (3.10), which are expressed by series [1]

$$
G\left(r, r^{\prime}\right)=\sum_{\alpha=0}^{\infty} U_{\alpha 0}(r) U_{\alpha 0}\left(r^{\prime}\right) / E_{\alpha 0}
$$

of unperturbed eigenfunctions $U_{\alpha 0}$ and eigenvalues $E_{\alpha 0}$. We note that such expansions are very complicated for practical solution of integral equations.

The solutions (4.1) $\varphi_{\alpha} U_{0 n L}$ at large distances are proportional to $U_{0 n L}$ or $\exp \left(-m \omega r^{2} / \hbar\right)$ and represent continuous functions. In this case, those solutions have derivatives of the order larger than the second and therefore they can be applied for semi-relativistic model of atomic nuclei [5]. In order to find relativistic corrections of eigenvalues, derivatives of the solution must be calculated till the fourth order. By using simple discretization methods this problem can be solved only very approximately because here derivatives are defined only till the second order.

The convergence conditions of eigenfunctions are presented in (4.5)-(4.6). These results are important for practical calculations of superheavy nucleus stability [7] and the energy spectrum of charmed and bottom mesons [6].

\section{References}

[1] F.W. Byron and R.W. Fuller. Mathematics of Classical and Quantum Physics. Dover Publications, New York, 1970.

[2] R.S. Guter and A.R. Janpolski. Differential Equations, High School. Moscow, 1976. (in Russian)

[3] A.J. Janavičius. Relativistic corrections in the average field of neutron and proton shells. Acta Physic Polonica B, 27:2195-2205, 1996.

[4] A.J. Janavičius. Green's functions and nonphysical solutions. Lithuanian Journal of Physics, 38(5):431-435, 1998.

[5] A.J. Janavičius. Shell stability of heaviest atomic nuclei in the semi-relativistic model. Lithuanian Journal of Physics, 41(3):232-236, 2001.

[6] A.J. Janavičius. Semi-relativistic model for charmed and bottom mesons. Proceedings of Scientific Seminar of the Faculty of Physics and Mathematics, pp. 35-45, 2005.

[7] A.J. Janavičius. Mathematical methods in the semi-relativistic single-particle models for superheavy nuclei. The 10th Worl Multi-Conference on Systemics, Cybernetics and Informatics, July 9-16, 2006, Orlando, Florida, USA: Proceedings, 3:66-68, 2006. 
[8] A.J. Janavičius and D.T. Jurgaitis. Integral equations for schrödinger equation in potential representation in the case of positive energies. Lithuanian Physics Collection, 25(3):33-38, 1985. (in Russian)

[9] A.J. Janavičius and D.T. Jurgaitis. Separation of scattering matrix with short range potential from background Coulomb field. Lithuanian Physics Collection, 26:273-278, 1986. (in Russian)

[10] A.J. Janawiczius and R. Planeta. A General Solution of the Schrödinger Equation in the Potential Representation. Krakow, Raport No. 1018/PL, 1978. (in Russian)

[11] N.A. Jelley. Fundamentals of Nuclear Physics. Cambridge University Press, New York, 1990.

[12] E. Merzbacher. Quantum Mechanics. New York, 1970.

[13] J.M. Ortega and V.G. Pool. An Introduction to Numerical Method for Differential Equations. Pitman Publishing Inc., New York, 1981.

[14] I.G. Petrovski. Lections about Theory Integral Equations. Science, Moscow, 1965. (in Russian)

[15] J.B. Zeldowich. Theory of perturbations for one dimension task of quantum mechanics. Journal of Experimental and Theoretical Physics, 31:1101-1105, 1985. (in Russian) 